\title{
Insight into Interfacial Behavior of Surfactants and Asphaltenes: Molecular Dynamics Simulation Study
}

\author{
Mohammad Ali Ahmadi ${ }^{{ }^{*}}$, Zhangxin Chen ${ }^{{ }^{*}}$ \\ 1) Department of Chemical and Petroleum Engineering, University of Calgary, Calgary, Alberta, Canada \\ ${ }^{*}$ Address to Corresponding Author: Department of Chemical and Petroleum Engineering, University of \\ Calgary, Calgary, Alberta, Canada. Email: mohammadali.ahmadi@ucalgary.ca, zhachen@ucalgary.ca TEL:
}

001-587-2889700

\section{Supplementary Information}

As demonstrated in Figures S1 (a) and (b), CTAB molecules were distributed mainly in the water phase and interacted more with water molecules (see Figures S1(a) and S1(b)). In this case, both dimers and trimers with the $\pi-\pi$ interactions were observed. The same behavior was also observed for CAPB for both island and archipelago architectures, as shown in Figures S1(e) and S1(f). The configuration of asphaltene molecules in the case of CAPB is mainly trimers with the $\pi-\pi$ interactions, as noted in Figures S1(e) and S1(f). In the case of TX-100, the configuration of the archipelago architecture is mainly dimers, and for the island architecture, a trimer is dominant, as depicted in Figures S1(c) and S1(d). It means that TX-100 has higher interaction with Archipelago asphaltene compared to island continental asphaltenes. In other words, TX-100 performs as a dispersant in contact with Archipelago than island asphaltenes. 


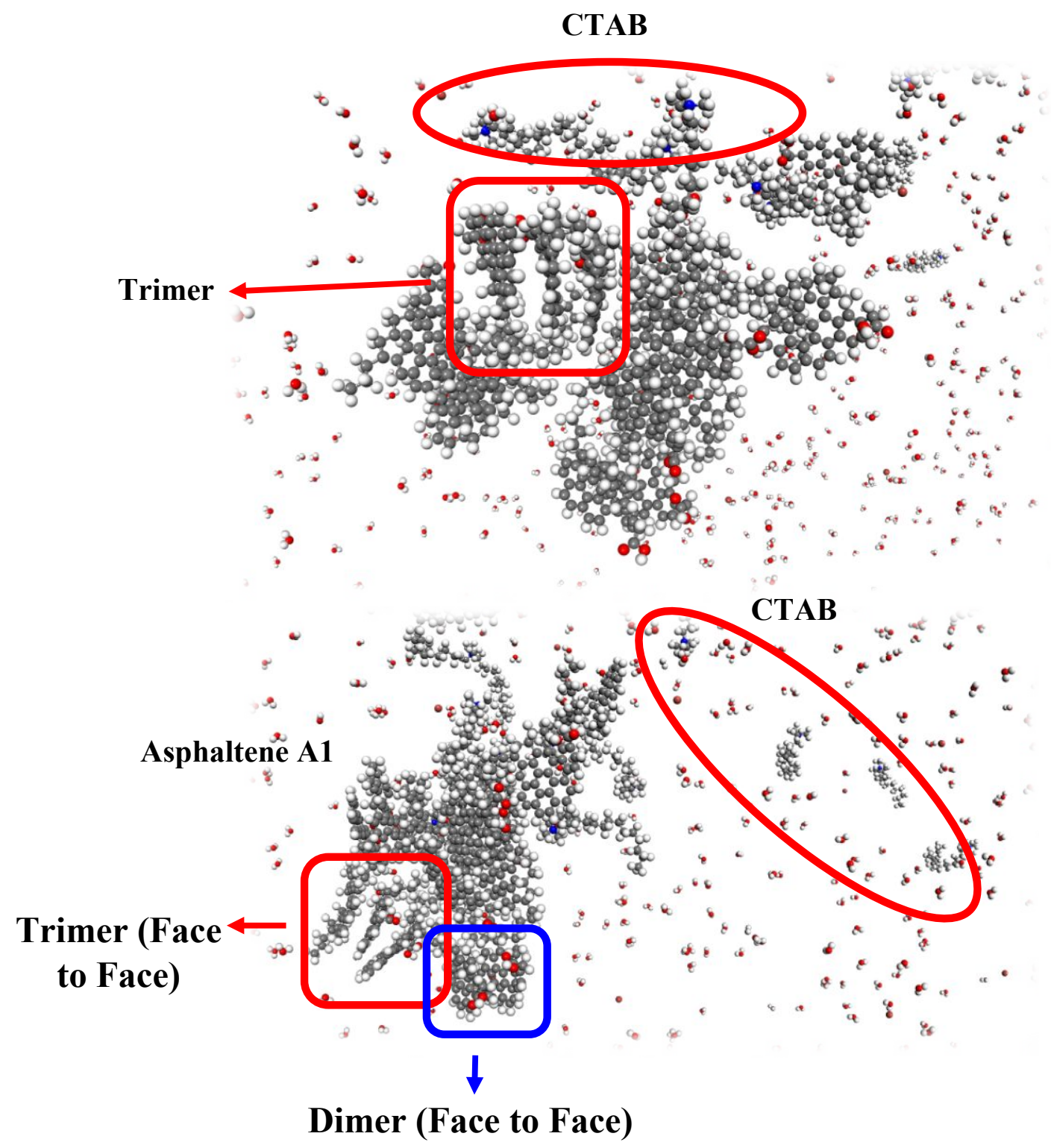

(a) 

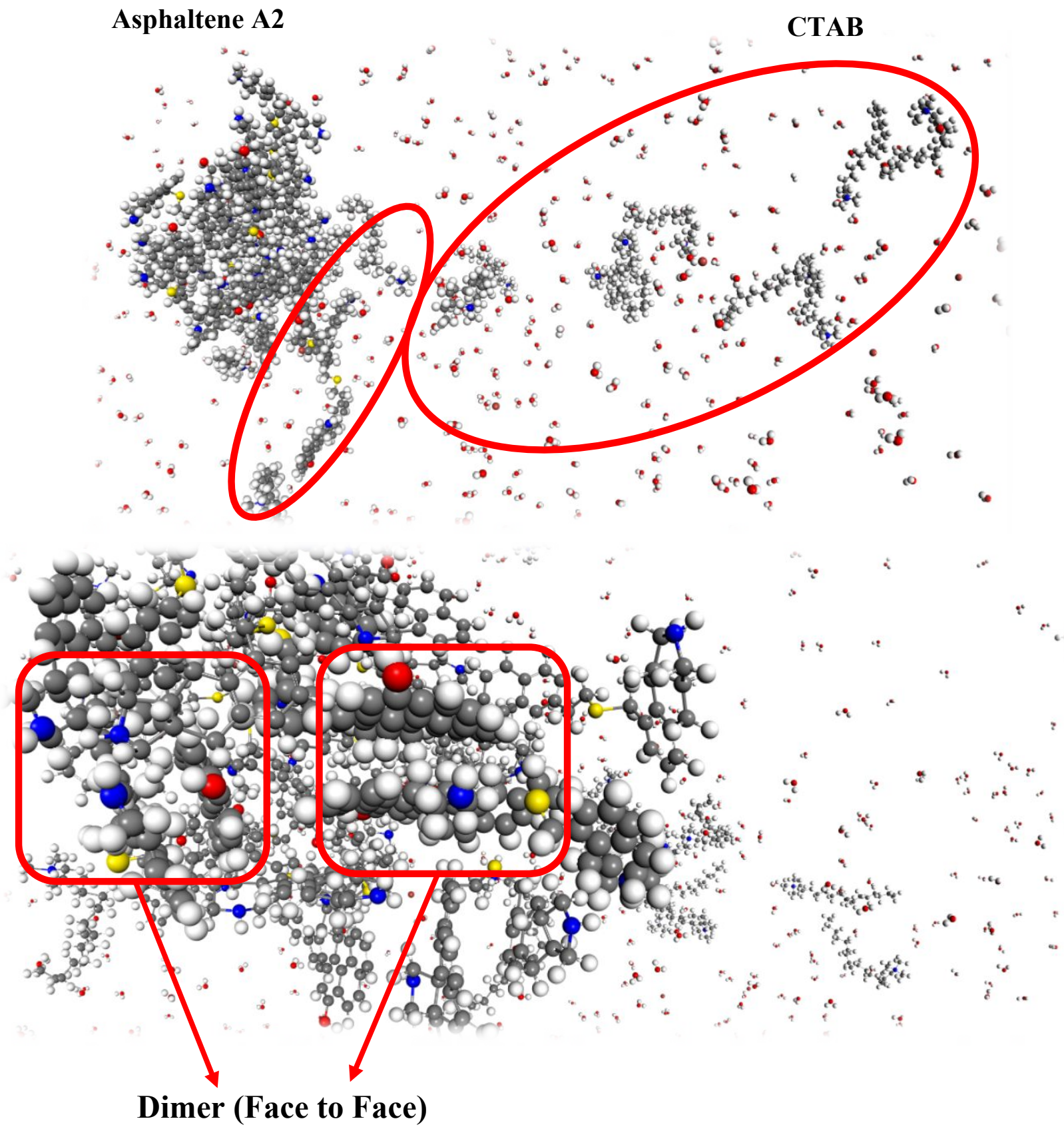

(b) 


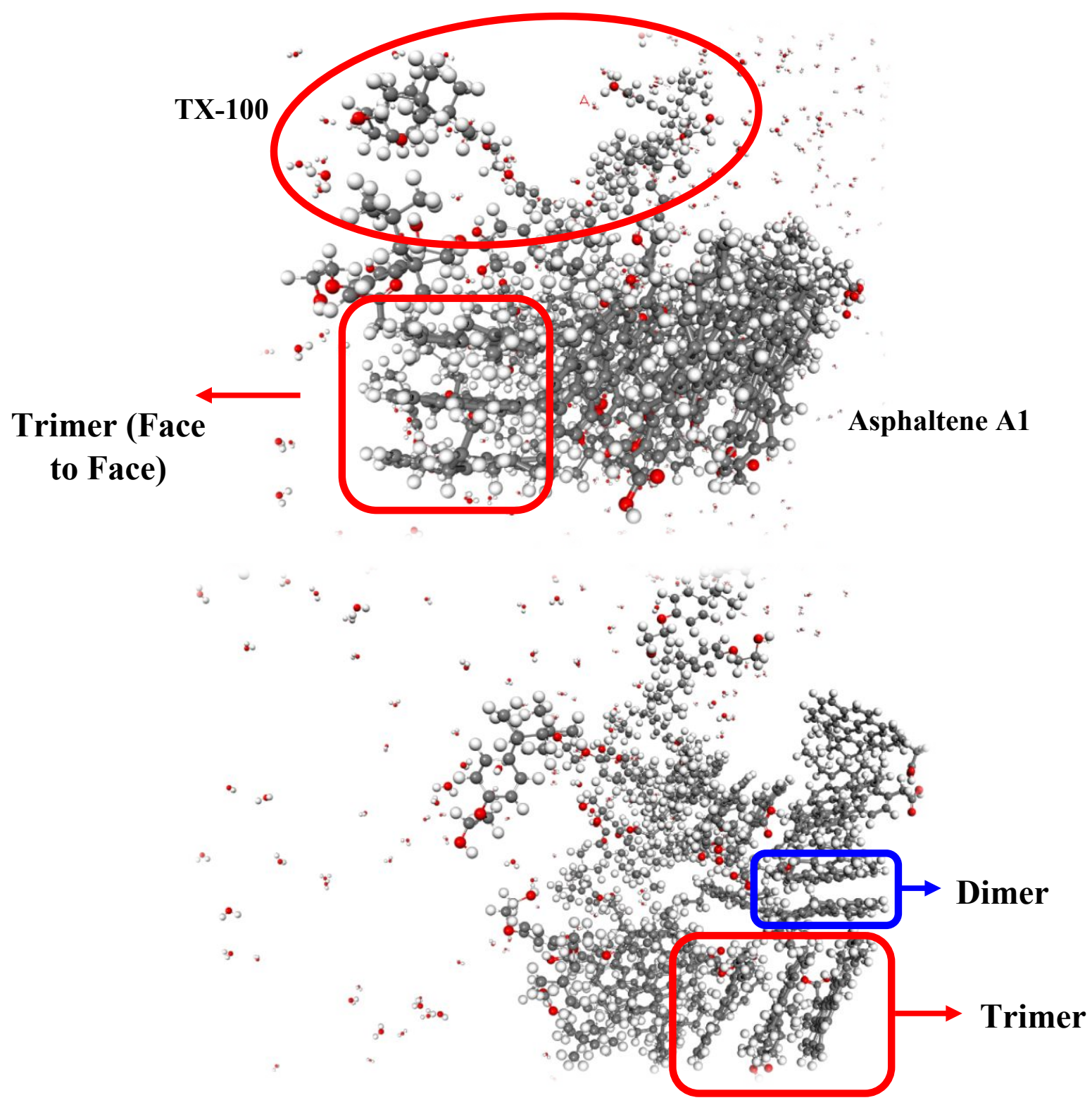

(c) 


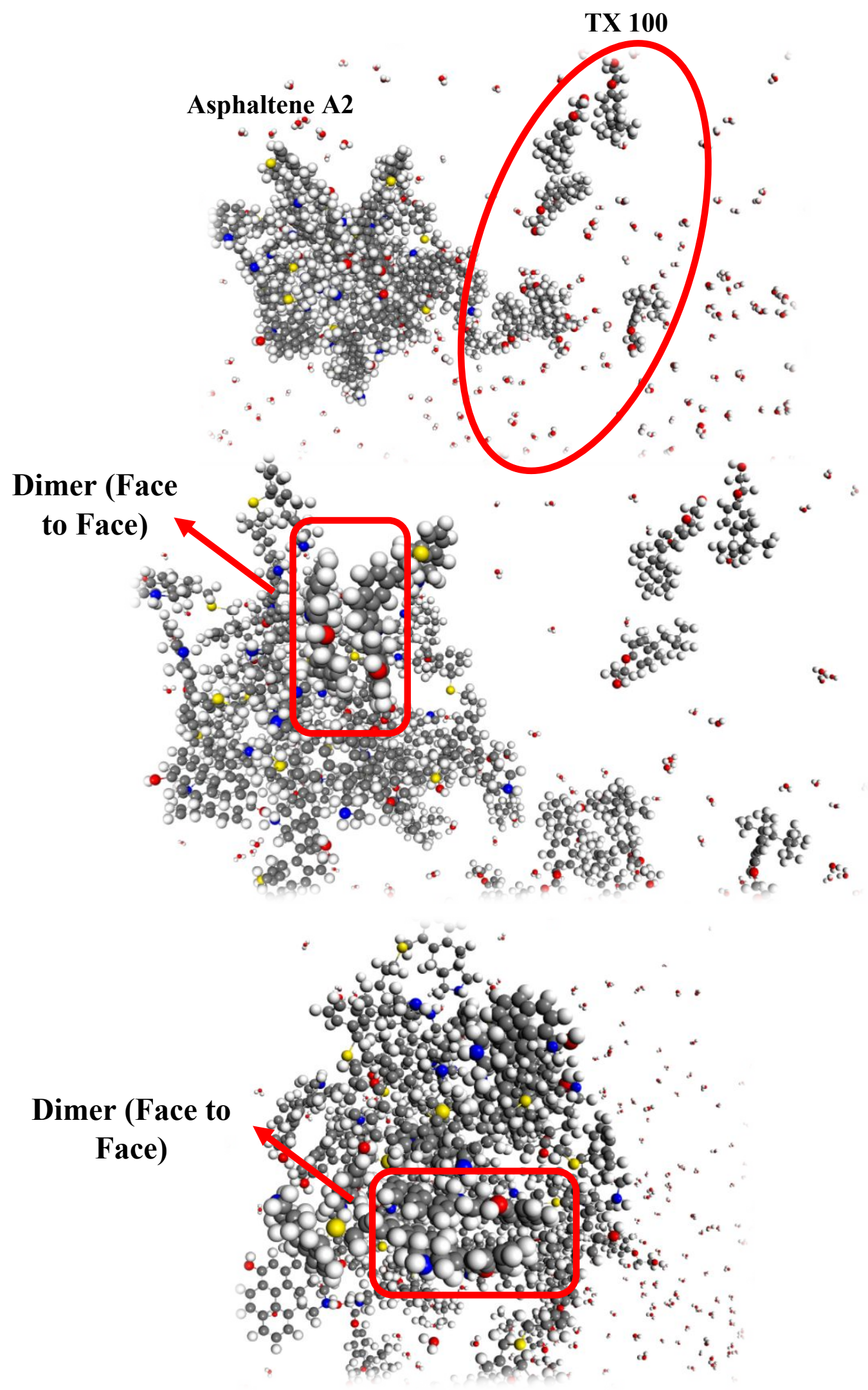

(d) 


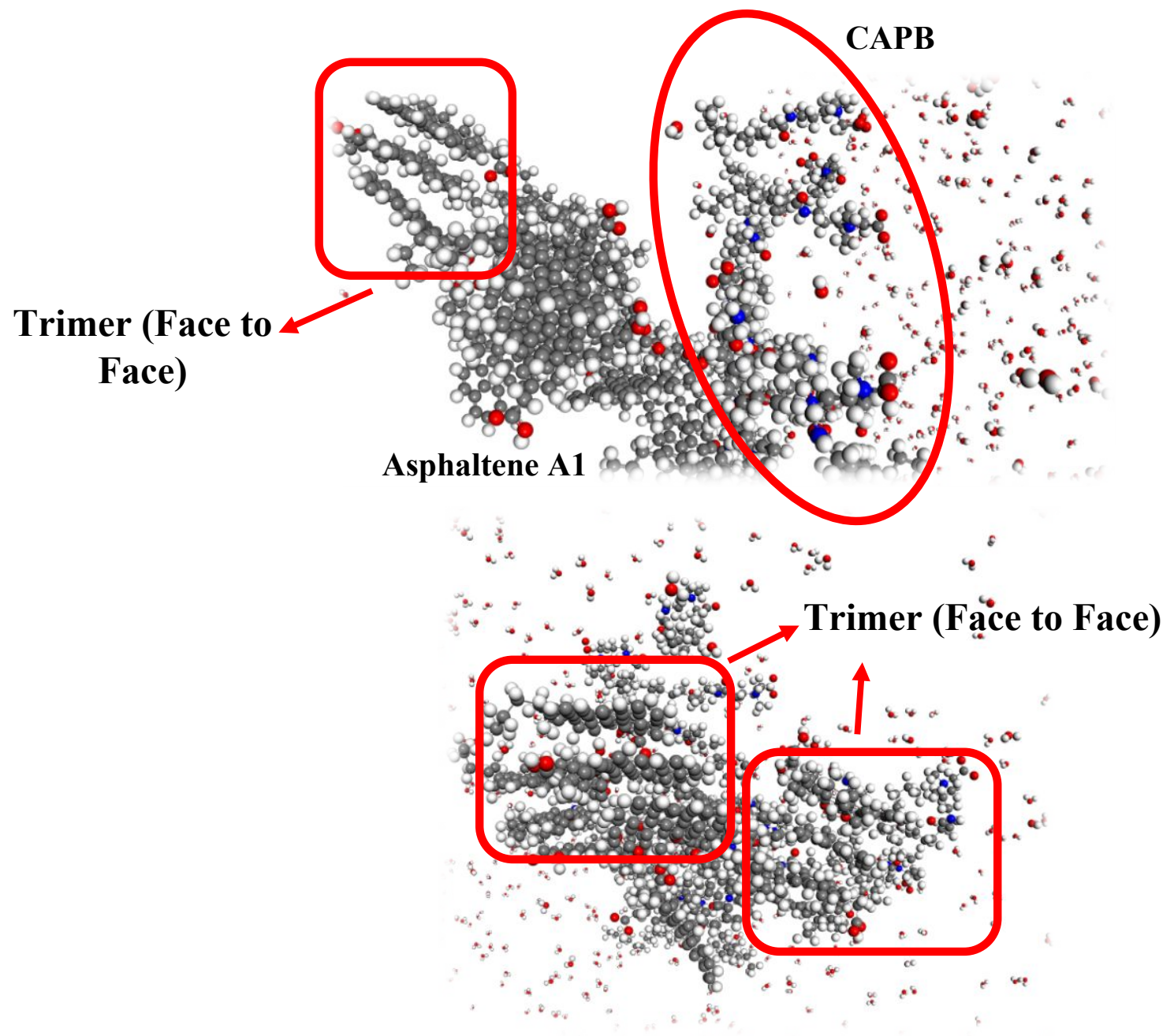

(e) 


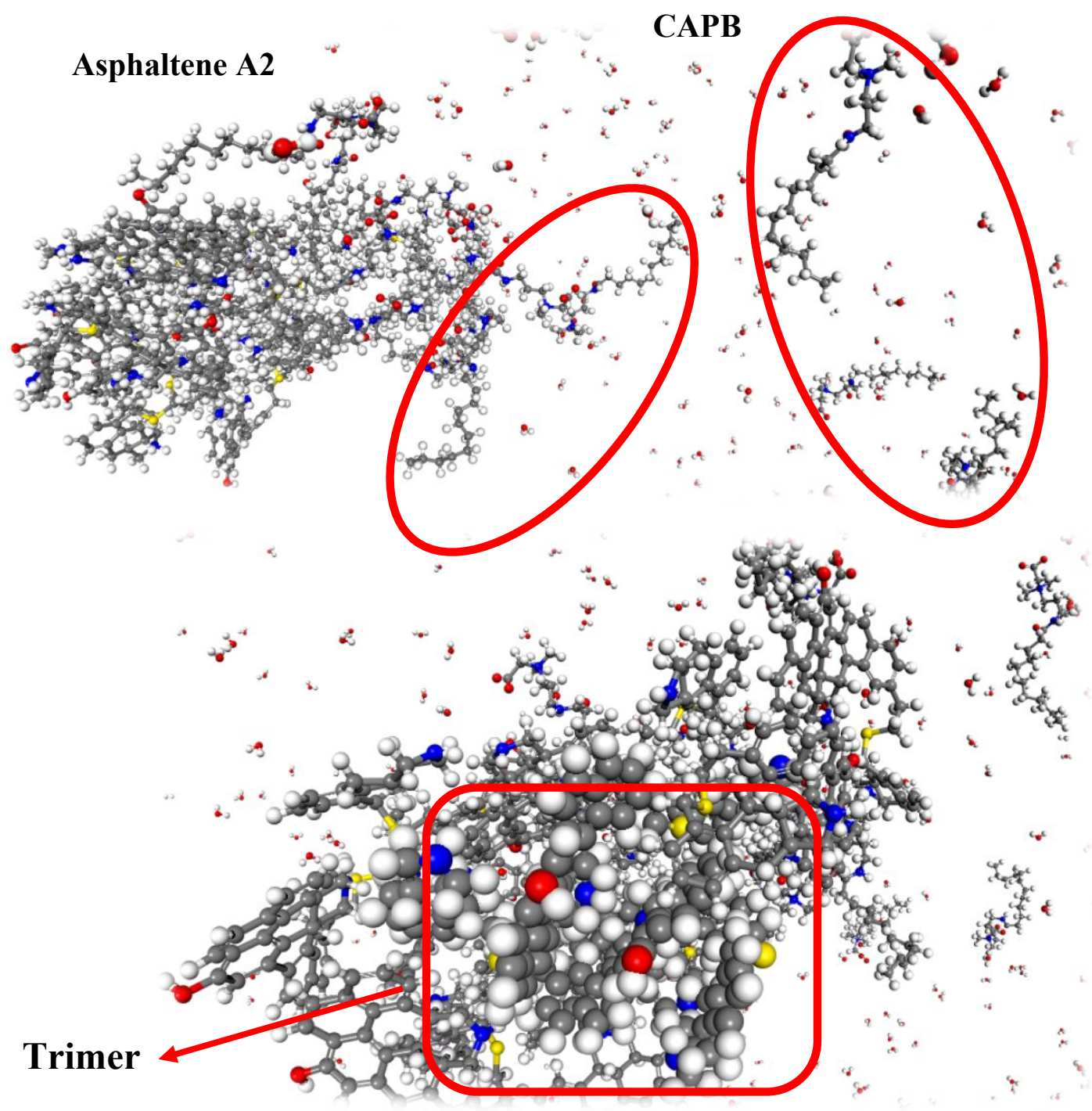

(f)

Figure S1. Snapshot of final configuration at 498K for a) CTAB-Asphaltene A1 b) CTABAsphaltene A2 c) TX-100-Asphaltene A1 d) TX-100-Asphaltene A2 e) CAPB-Asphaltene A1 f) CAPB-Asphaltene A2 